\title{
Direct Force Measurements during Transient Flow about Pitching Flat Plates
}

\author{
Huai-Te $\mathrm{Yu}^{1}$ \\ Chung Cheng Institute of Technology, National Defense University, 33551, Taoyuan, Taiwan, Republic of China \\ and \\ Luis P. Bernal ${ }^{2}$ \\ University of Michigan, Ann Arbor, Michigan, 48109
}

\begin{abstract}
This paper presents transient flow phenomena in terms of angles of attack by means of direct force measurement on pitching flat plate wings at a constant pitch acceleration. The wing motion is different from the most commonly used wing motion at constant pitch rates but generates comparable aerodynamics in a relatively short time. The wings were pitched from zero to maximum angles of attack ranging from 3 degrees to 42 degrees at mid-chord in a constant free stream Reynolds number of 8,900 . Three wing planform shapes with the same effective aspect ratio four are considered: rectangle, trapezoid, and triangle. Results show that the unsteady forces are developed following the similar trend as the maximum angle of attack is increased, giving a positive normal force and negative axial force extreme. The transient flow, occurring at the maximum angles of attack before steady state, gives oscillatory characteristics on both normal force and axial force coefficients, and further yields oscillatory lift and drag coefficients. The multiple extremes of positive normal force and negative axial force are in phase. These oscillation phenomena are more vigorous for rectangular and trapezoidal wings, especially at the angle of attack higher than 24 degrees, than for triangular wing. Two types of vortex dynamic system are further revealed from Strouhal number analysis.
\end{abstract}

\section{Nomenclature}

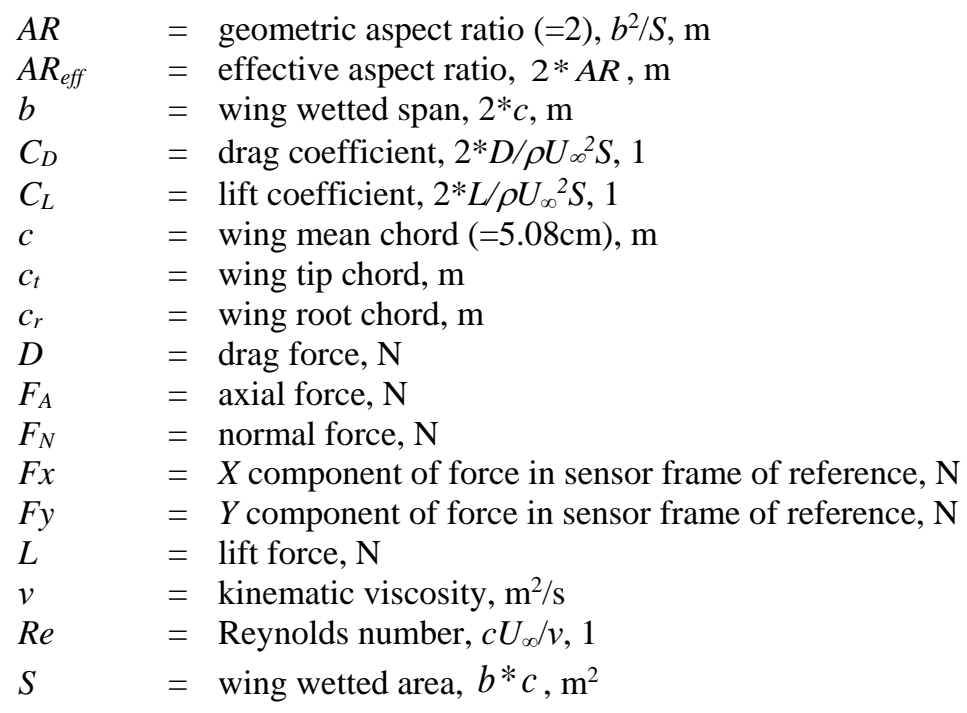

\footnotetext{
${ }^{1}$ Assistant Professor, Department of Mechanical and Aerospace Engineering, Chung Cheng Institute of Technology, National Defense University, htyu0504@ndu.edu.tw, and AIAA Member.

${ }^{2}$ Professor, Department of Aerospace Engineering, University of Michigan, 1pb@umich.edu, and AIAA Member.
} 
$t_{c} \quad=$ convective time, $c / U_{\infty}, \mathrm{s}$

$t_{p} \quad=$ pitch time, $\alpha_{m} / \alpha_{m}^{\prime}, \mathrm{s}$

$\alpha(t) \quad=$ angle of attack, degree

$\alpha_{m}^{\prime \prime} \quad=$ maximum pitch acceleration, degrees per second squared

$\alpha^{\prime}(t) \quad=$ pitch rate, degrees per second

$U_{\infty} \quad=$ constant free stream velocity, meter per second

\section{I.Introduction}

$\mathrm{T}$ he transient flow phenomena discussed in this paper is the unsteady flow that develops over pitching wings in a steady free stream. We consider the flow evolution from the initiation of the pitching motion, through the acceleration and deceleration phases of the wing motion, and during the flow relaxation to the steady state condition. We focus on pitching motions at constant acceleration/deceleration $\left( \pm 200 \% \mathrm{~s}^{2}\right)$ of rectangular, trapezoidal and triangular flat plate wings and final angles of attack in the range 3 to $42^{\circ}$.

The NATO Science and Technology Office AVT 202 panel report [1-5] discusses the flow characteristics for constant-rate pitching wing motions. A key feature is the development of large lift and drag forces during the motion, well above the steady state values for the corresponding angle of attack. A relaxation phase follows where forces decrease to the steady state value. Rotation rate effects account for the large forces during wing motion, often referring to the coupling effects of pitch rate and free stream velocity. Also, AVT 202 panel members documented the transient flow features of pitching rectangular flat plates at 45-degree angle of attack in free stream, and for translationally accelerated wings in still water, through multiple facilities. In addition to the large force increase during the wing motion, they reported local force maximum during the relaxation phase, which depends on reduced pitch rate $(K=$ $0.5 \mathrm{c} \alpha_{m}^{\prime} / U_{\infty}$ ). The local maximum appears at a phase around 7-8 convective time for $K$ equal to $\pi / 8$ (fast pitching case) and 10 convective times for $K$ equal to $\pi / 48$ (slow pitching case); the time was measured from the initiation of the motion. $\mathrm{Ol}$ and Babinsky [1], and Yu and Bernal [6] showed the dependence of this transient force increase during the relaxation phase on pivot axis location. As the time scale is shifted by the convective time required for a pivot axis location to travel from leading edge, the force maxima are found to be at the same phase for a given reduced pitch rate at a given maximum angles of attack [7]. Changes in force evolution because of flow acceleration in the initial phases has a little influence on the force maximum during the relaxation phase. Son et al. [5] discussed the formation of the local force maximum as a function of wing aspect ratio; they found that there is no maximum during the relaxation phase for aspect ratio equal to two.

In this study, we experimentally investigate the transient flow over pitching wings at several angles of attack at Reynolds number 8,900. The experiments were conducted in a water channel facility, using direct force measurement. Three wing planforms are considered: rectangle, trapezoid, and triangle. All wings have the same mean geometric aspect ratio equal to two. In order to reduce the inertial contribution to the measured force, the wings were pitched from zero to a fixed angle of attack about the mid-chord at the same constant acceleration/deceleration. The main goal of this study is to determine the transient of unsteady flow as a function of angle of attack at the end of the wing motion and wing planform shape.

\section{II.Experimental Setup}

\section{A. Water Channel}

The low-turbulence free-surface water channel at the University of Michigan was employed. A steady freestream velocity was produced through the control of $\mathrm{AC}$ induction motor and water depth. The experiment was conducted in an area of $61 \mathrm{~cm}$ wide and $57 \mathrm{~cm}$ deep. In this study, the freestream velocity is $17.5 \mathrm{~cm} / \mathrm{s}$, corresponding to chordbased Reynolds number of 8,900 . The room temperature was kept at $72{ }^{\circ} \mathrm{F}$.

\section{B. Wing Configuration}

The wings are flat plates with three different wing planforms, as shown in Fig. 1. All mean chord lengths are two inches and the spans are four-inches. The free surface of water channel serves as a symmetry plane which would result in an effective aspect ratio of four for all wing planforms considered. All wings were made of the clear acrylic sheet with the same thickness of 0.125 inches. The edges are rounded with a diameter of curvature $6.25 \%$ of the mean chord. The wing planform is symmetric about the mid-chord line which is also the pitch axis of the wing motion. The 
geometric parameters of the wings are given in Table 1. The sweep angles of the wings, defined by the half-chord line, are all zero.

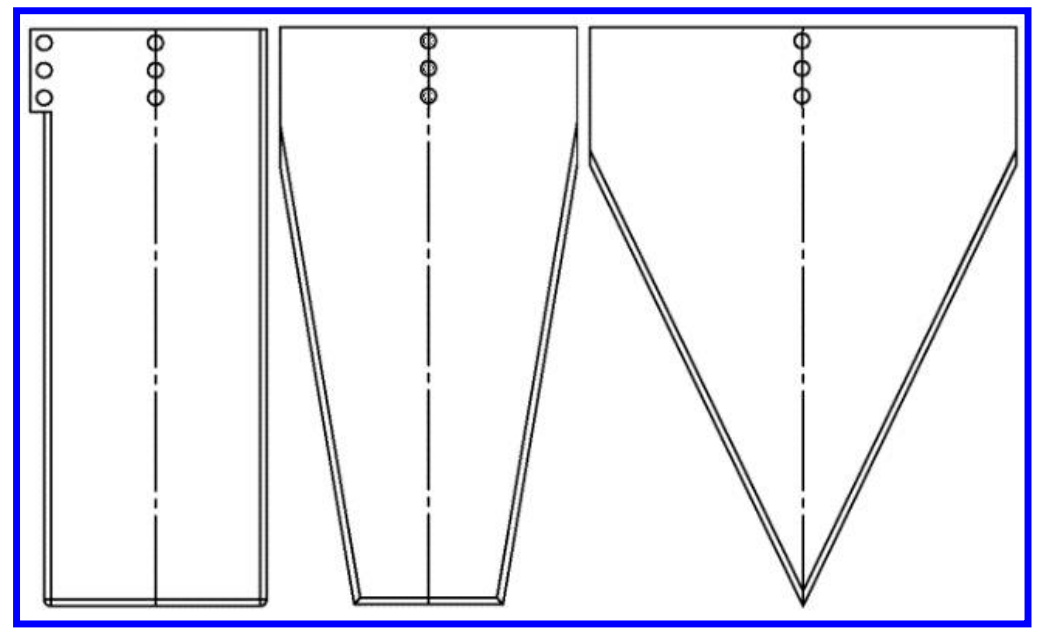

Fig 1 Wing planform of interest

Table 1 Wing geometry

\begin{tabular}{cccccccc}
\hline Properties & Mass, g & $\begin{array}{c}\text { Mean chord, } \\
\text { inches }\end{array}$ & $\begin{array}{c}\text { Leading-edge } \\
\text { sweep angle, } \\
\text { degree }\end{array}$ & $\begin{array}{c}\text { Root } \\
\text { chord, } \\
\text { inches }\end{array}$ & $\begin{array}{c}\text { Tip } \\
\text { chord, } \\
\text { inches }\end{array}$ & $\begin{array}{c}\text { Taper ratio, } \\
c_{t} / c_{r}\end{array}$ & $\begin{array}{c}\text { Sweep angle (with } \\
\text { reference to half } \\
\text { chord line), degree }\end{array}$ \\
\hline Rectangle & 22.9 & 2 & 0 & 2 & 2 & 1 & 0 \\
\hline Trapezoid & 24.1 & 2 & 9.46 & 2.67 & 1.33 & 0.5 & 0 \\
\hline Triangle & 27.7 & 2 & 26.57 & 4 & 0 & 0 & 0 \\
\hline \hline
\end{tabular}

\section{Wing Kinematics}

The wing motion considered in this study is a quadratic time variation of angles of attack from $0^{\circ}$ to several maximum pitch angles $\left(\alpha_{m}\right)$. An example of motion evolution is illustrated in Fig. 2a. The maximum pitch angle ranges from three degrees to 42 degrees with a step increment of three degrees. As a result, the total number of force traces is 14 for each wing planform, corresponding to 14 maximum pitch angles. As shown in Fig. 2b, the present wing motion yields a pitch speed $\left(\alpha^{\prime}\right)$ linearly changing with time. However, the $\alpha_{m}$ is nonlinearly dependent on corresponding pitch time $\left(t_{p}\right)$ because of a constant value of pitch acceleration $\left(\alpha^{\prime \prime}{ }_{m}\right) 200 \mathrm{deg} / \mathrm{s}^{2}$ being commanded. These characteristics are featured in Fig. 2c-d. Additionally, a maximum pitch speed $\left(\alpha_{m}^{\prime}\right)$ of $100 \mathrm{deg} / \mathrm{s}$ and start/stop pitch speed $\left(\alpha^{\prime}\right)$ of $2.25 \mathrm{deg} / \mathrm{s}$ were specified for the rotary table motion controller. The motion of the rotary table is formulated by Eq. (1)-(2).

$$
\begin{aligned}
& \alpha(t)=\left\{\begin{array}{c}
\alpha_{0}^{\prime} t+\alpha_{m}^{\prime \prime} t^{2} / 2,0 \leq t \leq t_{p} / 2 \\
\left(\alpha_{0}^{\prime}+\alpha_{m}^{\prime \prime} t_{p} / 2\right)\left(t-t_{p} / 2\right)+\left(\alpha_{m}^{\prime \prime} / 2\right)\left(t-t_{p} / 2\right)^{2}+\alpha_{m} / 2, t_{p} / 2 \leq t \leq t_{p}
\end{array}\right. \\
& \alpha^{\prime}(t)=\left\{\begin{array}{c}
\alpha_{0}^{\prime}+\alpha_{m}^{\prime \prime} t, 0 \leq t \leq t_{p} / 2 \\
\alpha_{0}^{\prime}-\alpha_{m}^{\prime \prime}\left(t-t_{p}\right), t_{p} / 2 \leq t \leq t_{p}
\end{array}\right.
\end{aligned}
$$

The wing kinematics were implemented using a motorized rotary table B4818TS by Velmex Inc. with a step motor operating in half step mode, giving 20 steps per degree and 200 degrees-per-second maximum pitch speed. After reaching $\alpha_{m}$, the wing motion was paused for 40 seconds to capture the transient flow, which corresponds to 137 convective times. Then the motion was inverted to return the wing to the initial zero angle of attack. The wing was held at the zero angle of attack for at least 31 convective times to allow flow disturbances introduced by the wing motion to dissipate. For each case, the wing motion was repeated 60 times and the results phased averaged.

The dimensionless parameter $K$ defined in Eq. (3) is commonly employed for pitching wings/airfoils undergoing constant pitch rate; this parameter is called reduced pitch rate. For present pitch wings with a constant rate of change of pitch rate, the reduced pitch rate is still useful as if it is expressed as a function of maximum pitch angle and the 
time of disturbance with respect to free stream convection. As listed in Table 2, present test configuration gives the reduced pitch rate ranging from 0.034 to 0.119 .

$$
K=0.5 c \alpha_{\mathrm{m}}^{\prime} / U_{\infty}=0.5 \alpha_{m}\left(t_{c} / t_{p}\right)
$$

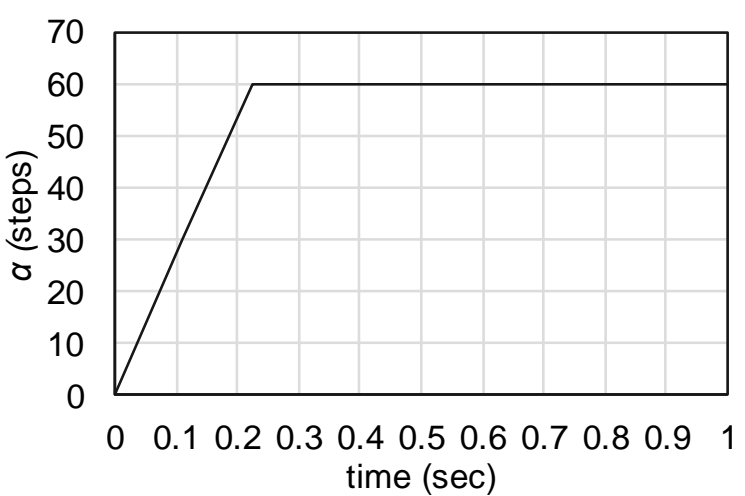

(a) An example of the time evolution of angle of attack for a maximum angle of attack of 3 degrees (60 steps)

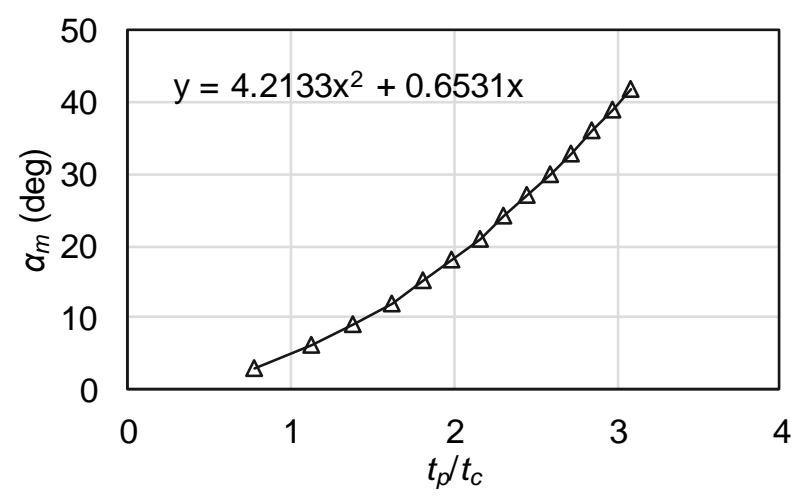

(c) Maximum pitch angle as a function of time

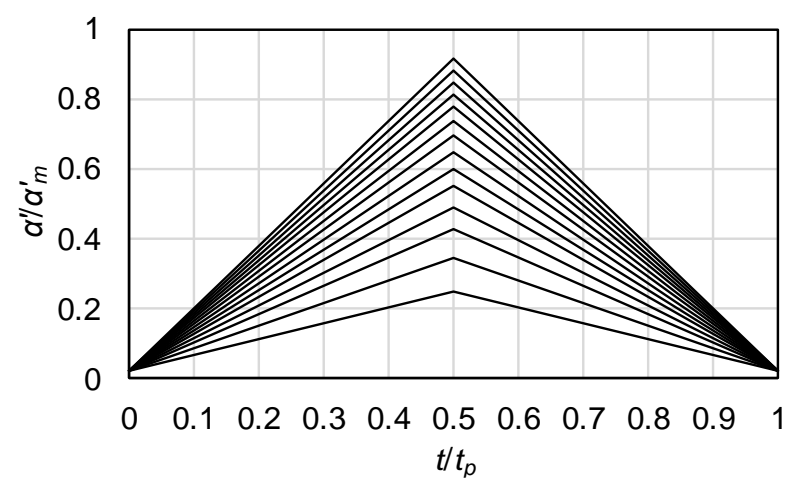

(b) Scaled pitch rates as a function of pitch time

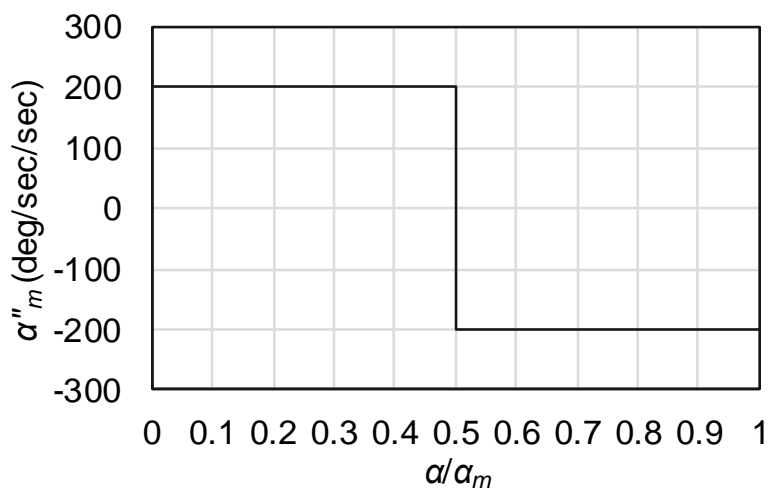

(d) Pitch acceleration as a function of pitch angle

Fig. 2 Characteristics of wing kinematics of interest.

Table 2 Parameters for Wing Kinematics

\begin{tabular}{ccccccccccccccc}
\hline \hline AOA & $3^{\circ}$ & $6^{\circ}$ & $9^{\circ}$ & $12^{\circ}$ & $15^{\circ}$ & $18^{\circ}$ & $21^{\circ}$ & $24^{\circ}$ & $27^{\circ}$ & $30^{\circ}$ & $33^{\circ}$ & $36^{\circ}$ & $39^{\circ}$ & $42^{\circ}$ \\
\hline$t_{p \_ \text {theo }}$ & 0.223 & 0.325 & 0.402 & 0.468 & 0.526 & 0.578 & 0.626 & 0.671 & 0.713 & 0.752 & 0.790 & 0.826 & 0.861 & 0.894 \\
\hline$t_{p \_ \text {exp }}$ & 0.224 & 0.325 & 0.403 & 0.469 & 0.527 & 0.579 & 0.627 & 0.672 & 0.714 & 0.753 & 0.791 & 0.827 & 0.862 & 0.895 \\
\hline$t_{p} / t_{c}$ & 0.770 & 1.118 & 1.386 & 1.612 & 1.811 & 1.991 & 2.156 & 2.310 & 2.455 & 2.592 & 2.722 & 2.847 & 2.966 & 3.081 \\
\hline$K$ & 0.034 & 0.047 & 0.057 & 0.065 & 0.072 & 0.079 & 0.085 & 0.091 & 0.096 & 0.101 & 0.106 & 0.110 & 0.115 & 0.119 \\
\hline \hline
\end{tabular}

\section{Direct Force Measurement and Data Processing}

The wing was mounted on the water channel by fastening it to a force sensor through a sensor adapter plate and a rod clamped to the rotary table (see [8] for a description of the installation). The force sensor and rotary table were mounted on a motorized traverse above water channel free surface. The position of the three-hole pattern on the wing, as shown in Fig. 1, was designed to align force sensor axis with the rotary table axis. The force sensor was a Nano 43 Force/Torque sensor manufactured by ATI Industrial Automation. All six components of force and torque are measured in a Cartesian coordinate system with axes normal to the wing, along with the wing chord and along the wing span. The sensor adapter was made of aluminum and designed to minimize its contribution to the inertia of the measurement system. The total mass attached to the sensor (including the wing itself, the sensor adapter and screws) is no more than 46.2 grams, which makes the inertia negligible compared to the hydrodynamic forces. 
Processing of force data includes an application of low-pass filter and a tare procedure. In order to avoid phase distortion, to reduce spurious oscillations introduced by the filter, and to provide sufficient noise attenuation, the lowpass digital filter used was a zero-phase first-order two-path Butterworth filter. The cutoff frequency of the filter was chosen to be $8.7607 \mathrm{~Hz}$. The noise introduced by the rotary table and the stepper motor is in a range of $100 \mathrm{~Hz}$ to $600 \mathrm{~Hz}$, and the wing vibration fundamental frequency in air and in still water is $50 \mathrm{~Hz}$ and $15 \mathrm{~Hz}$, respectively, which are significantly higher than the filter cutoff frequency.

The tare procedure includes static tares and dynamic tares to isolate the hydrodynamic force from model inertia and model weight contributions to the measurement force. The static tares are measurements in air and still water at fixed angle from $0^{\circ}$ to maximum angle of attack 45 degrees with an increment of 3 degrees. The dynamic tares are measurements in air with the same kinematics as in the flow experiments. In addition, the force measurements at fixed angle of attack in steady flow are also considered. The kinematics is similar to static tare measurements but with a longer hold duration at the free-stream velocity of interest. In all force measurements, the same filter cutoff frequency was used.

All force measurements were repeated 60 times from $0^{\circ}$ to a fixed maximum angle of attack with a sampling rate of $5,000 \mathrm{~Hz}$, and ensemble averages are reported. It is found that the present wing configuration yields negligible static tares because of the very small mass of the wing and mounting hardware.

\section{III.Results and Discussion}

This chapter is structured as follows. The first three sections discuss the overlook of force evolution as a function of time for rectangular, trapezoidal, and triangular flat plates, successively. Followed by the comparison of instant force at given maximum angles of attack before relaxation process with the steady force, among various wing planforms. The last section discusses the transient oscillatory forces during the relaxation process before steady state.

\section{A. Force evolution of rectangular wing}

Figures 3 and 4 show the plate normal and axial (along with the chord) force during the transient for the rectangular wing. The vertical axes are normalized as force coefficients in the classical form, the horizontal axes are the time normalized by the convective time $\left(c / U_{\infty}\right)$. The cross marker represents measured force as the wing instantly stops at a maximum angle of attack. The marker is used to distinguish unsteady force by a pitching wing and transient force at a given maximum angle of attack. In the following figures, the similar cross marker is used.

In Fig. 3, several features are noted. First, the initial acceleration and deceleration do not show the peaks found for the linear ramp motion considered in AVT 202. Here the acceleration is significantly less and does not result in significant apparent mass spikes in the force signal. Second, as the maximum angle of attack is increased the force time evolution follows the same trend before the occurrence of force saturation during wing motion. This is striking because rotation rate effects that generally scale with pitching rate increase considerably as the maximum pitch angle is increased in a free stream. Third, the transient after the wing motion stops varies considerably depending on the maximum pitch angle. For a small maximum angle of attack in the range 3 - 9 degrees, the normal force reaches a maximum within the wing motion and then decreases rapidly even the wing stops, and then followed by a gradual approach (increase) to the steady state value. For these maximum angles of attack at steady state, there is a large increase of normal force with the angle of attack. For the maximum angle of attack in the range $12-21$ degrees, a second maximum develops around 3.8 convective times, being little dependent on the maximum angle of attack of interest. The second maxima reach the highest values in the course. After the second maxima, the normal force monotonically approaches (decreases) steady state value. For the maximum angle of attack in the range 24 - 36 degrees, multiple maxima develop at a later time. The third maxima develop at the convective time less than 10 . Other local maxima follow at convective times of $\sim 12, \sim 17$ and $\sim 22$. Finally, for the maximum angle of attack of 39 and 42 , the first two maxima merge and a second maximum is observed at 10 convective times. This latter case is very similar to the AVT 202 case, which was for the maximum angle of attack of 45 degrees and very different wing motion kinematics.

Figure 4 shows the force component in the chord direction as a function of time. Similar to Fig. 3 the results are plotted in the normalized form: axial force coefficient. Several features are noted. First, the axial force is low except for large angles of attack. This is expected and suggests that pressure force dominates the hydrodynamic force on the wing. The second important feature is the development of large leading edge suction at about 3 convective times. The positive axial force is defined toward the trailing edge. The growth of leading edge suction peak reaches its minimum 
value before the wing motion stops. The significant negative values at the same time as the large normal force indicate that the flow about the leading edge is attached. And there is a significant leading edge suction associated with the high-speed flow at leading edge. Furthermore, the axial force oscillation at 10 convective times and greater indicates a vortex shedding process during the transient, which disappears at steady state. This vortex shedding process affects the leading-edge flow and consequently the axial force on the wing.

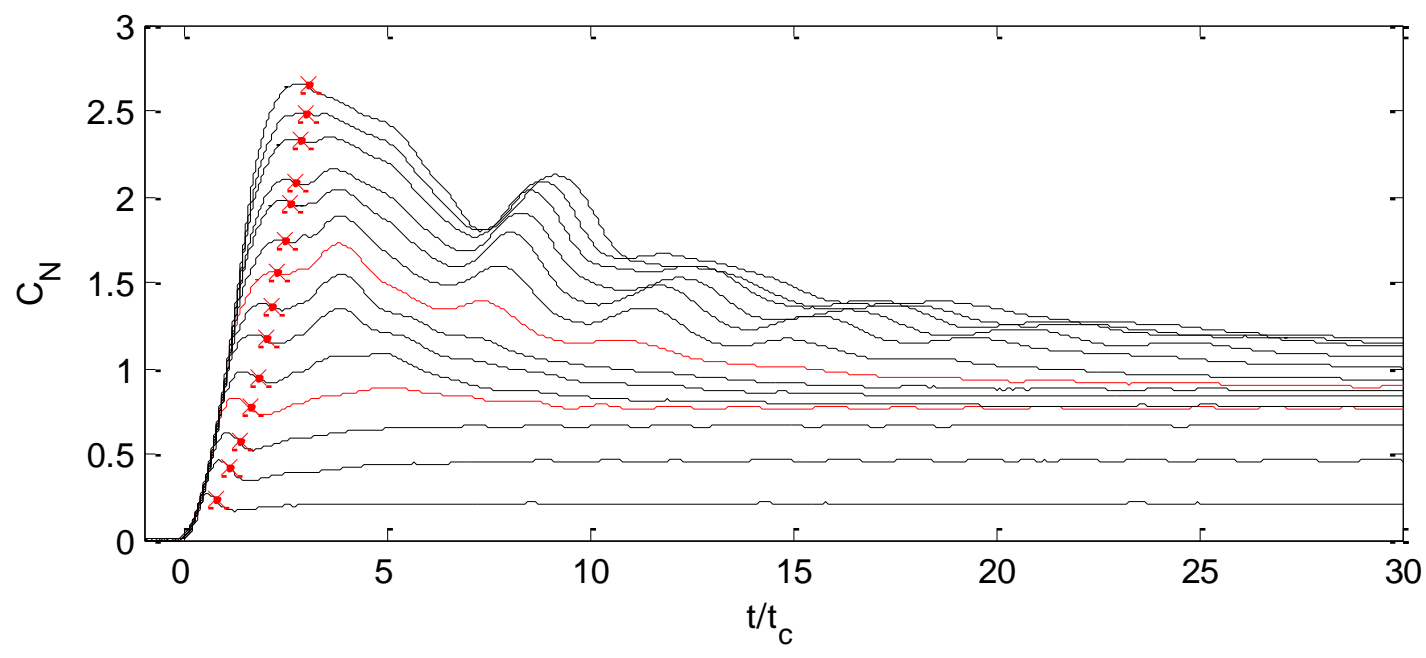

Fig. 3 Plate normal force coefficient of a rectangular plate as a function of normalized time for the maximum angle of attack in the range $3-42$ degrees in step increments of 3 degrees.

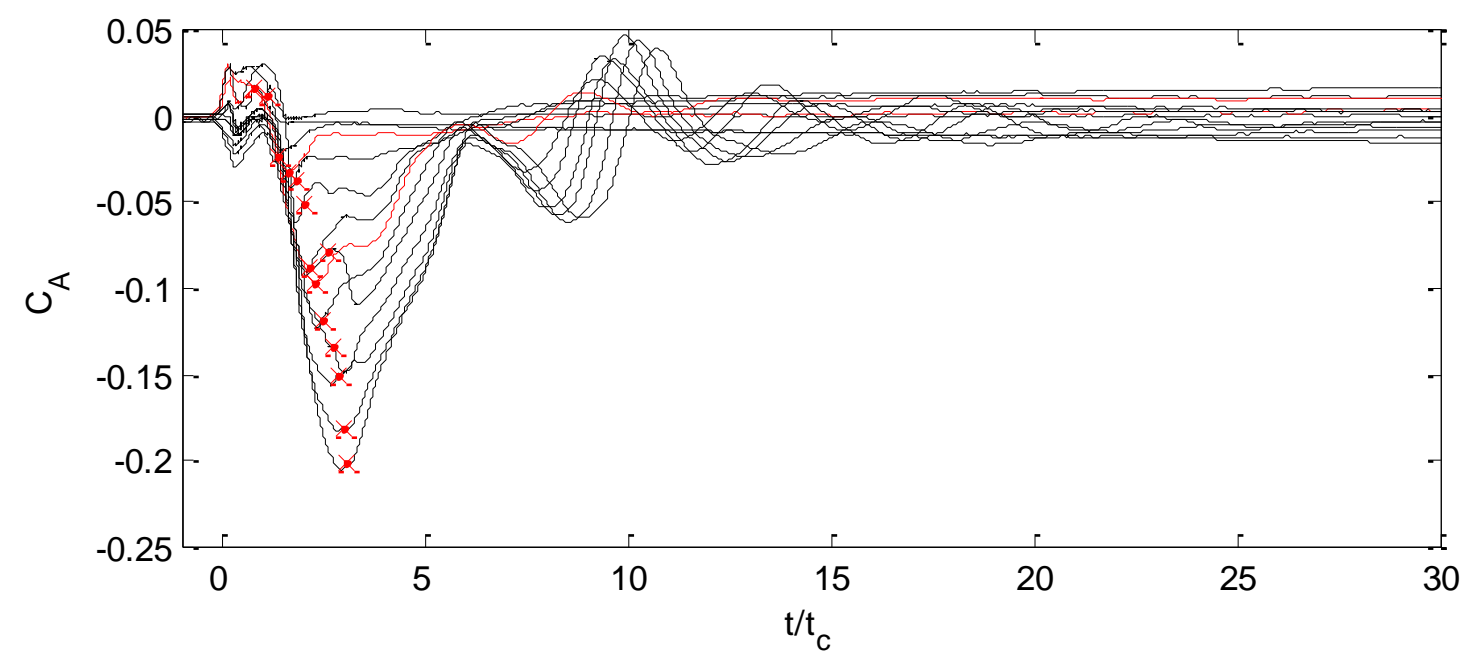

Fig. 4 Axial force coefficient of a rectangular plate as a function of normalized time for maximum angles of attack in the range $3-42$ in step increments of 3 degrees.

Figures 5 and 6, for the rectangular wing, show the evolution of lift and drag coefficients, respectively, as a function of time at all angles of attack considered. The features of lift coefficient are similar to the results of normal force coefficient. Because of leading edge suction, the maxima of lift coefficient during the wing motion are more significant than that of normal force coefficient. The lift coefficient at the maximum is very large for the large maximum angle of attack, more than a factor of two compared to the steady state values at 30 convective times. Unlike the spike-like course of lift coefficient before the wing stops, the drag coefficient increases rapidly to the maximum angle of attack. Clearly, the transient vortex shedding impacts the lift and drag coefficients, resulting in lift coefficient fluctuations of the order of 0.5 . 


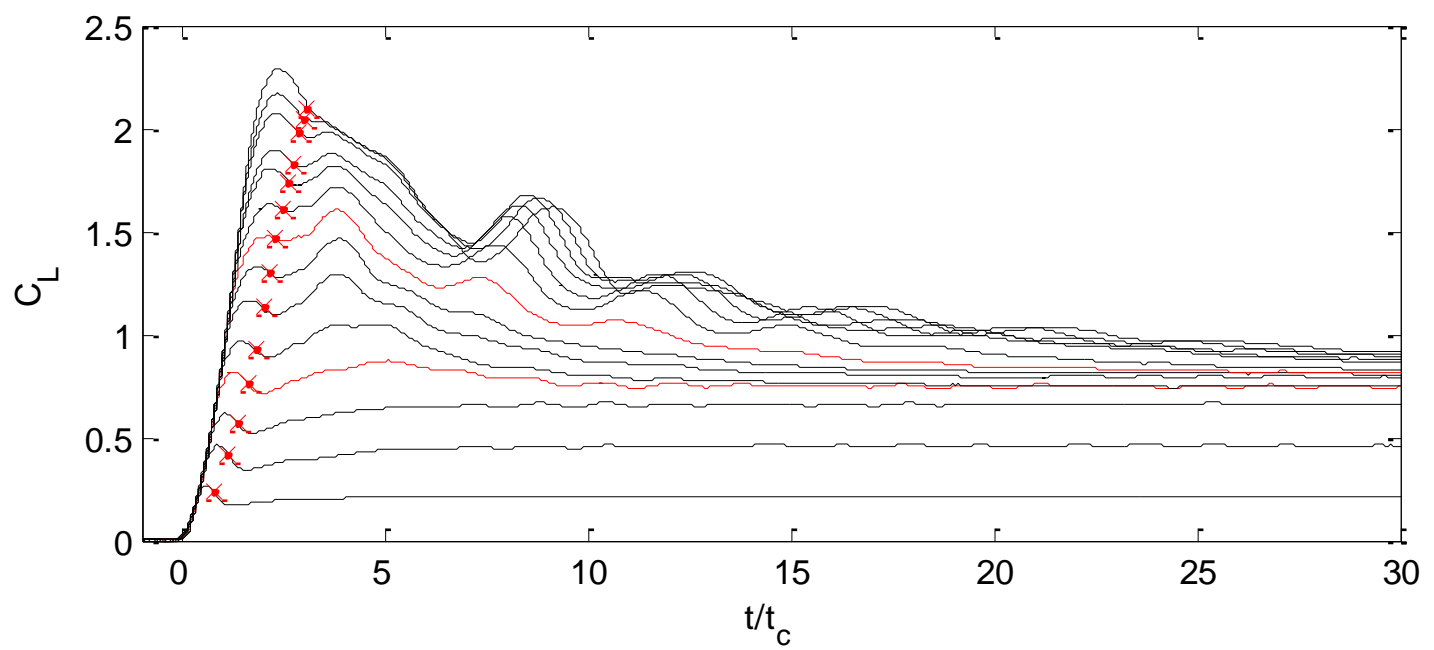

Fig. 5 Lift coefficient of a rectangular plate as a function of normalized time for maximum angles of attack in the range $3-42$ in step increments of 3 degrees.

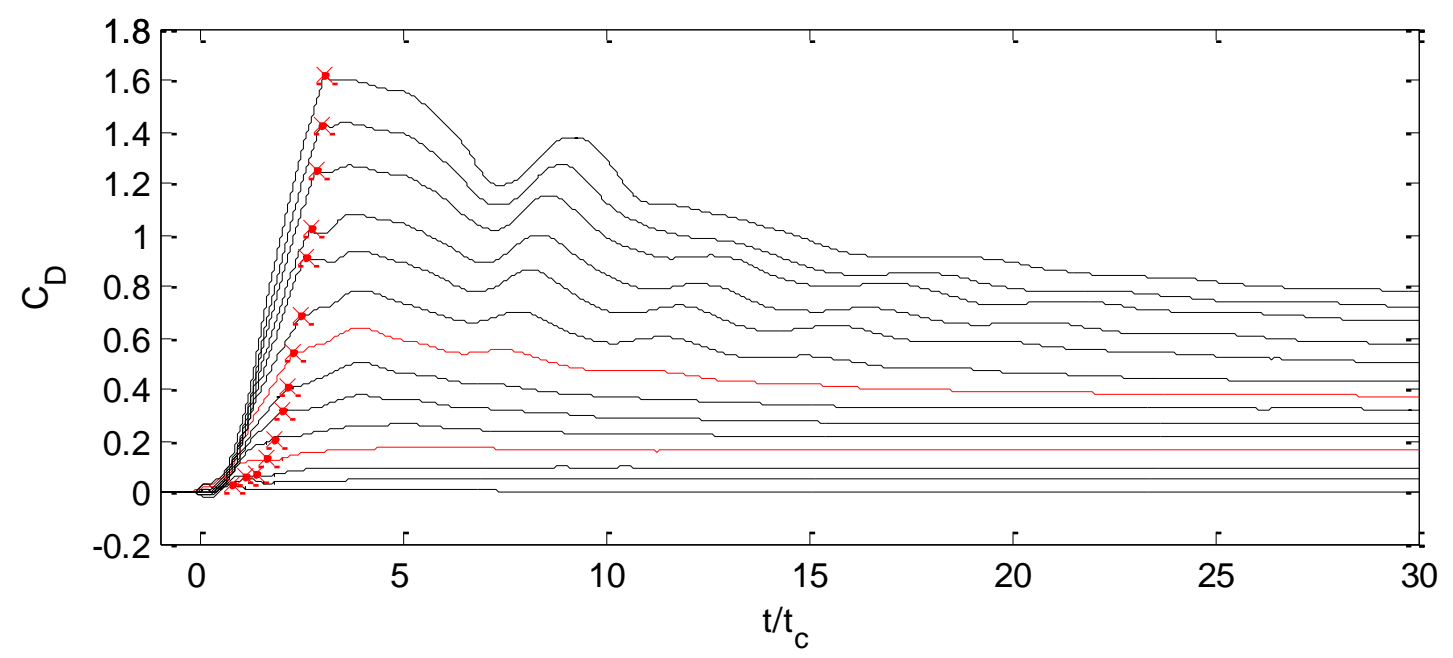

Fig. 6 Drag coefficient of a rectangular plate as a function of normalized time for maximum angles of attack in the range $3-42$ in step increments of 3 degrees.

\section{B. Force evolution of trapezoidal wing}

Figures 7 through 10, for the trapezoidal wing, show the evolutions of the plate normal, axial, lift and drag coefficients as a function of time normalized by convective time, successively. These data show substantially the same features as for the rectangular wing. The similar breakdown of flow regimes is observed. As shown in Fig. 7, for instance, the maximum angle of attack in the range 3-12 degrees produces the rapid increase of normal force coefficient within the wing motion, causing the first local maximum. The first local maximum is in phase with the negative local minimum axial force coefficient, as shown in Fig. 8, similar to that of the rectangular wing. Following a decrease, a gradual increase is present toward a steady state. For a maximum angle of attack in the range $15-24$ degrees, the development of a second local maximum around 3.8 convective times is revealed, similar to that of the rectangular wing. The second local maximum has less dependence on the maximum angle of attack and grows higher than the first maximum. The third maximum appears after 5 convective times, varying according to the maximum angle of attack. Following the third maximum is a slow decrease in transition toward a steady state. Interestingly, the third local maximum is not observed for the maximum angle of attack of 24-degree; the normal force coefficient decreases gradually after the second local maximum. For a maximum angle of attack in the range $27-39$ degrees, the first and second maxima of normal force coefficient are present and the second maxima remain higher than the first 
maxima. The third maximum appears at a later time around 10 convective times. For 42-degree maximum angle of attack, however, the value of the first maximum becomes higher than that of the second maximum. The third maximum appears around 10 convective times similar to the lower maximum angles of attack 30-39 degrees. The transient maxima of the trapezoidal wing are not as strong as that of the rectangular wing. These maxima are also associated with the negative minimum axial force coefficients, implying the significant development of leading edge suction and the transient vortex shedding process like that of the rectangular wing. At the steady state of 30 convective times, the normal force coefficient increases rapidly with the maximum angles of attack below 12 degrees. At a higher maximum angle of attack, the increase of normal force coefficient becomes slowly.

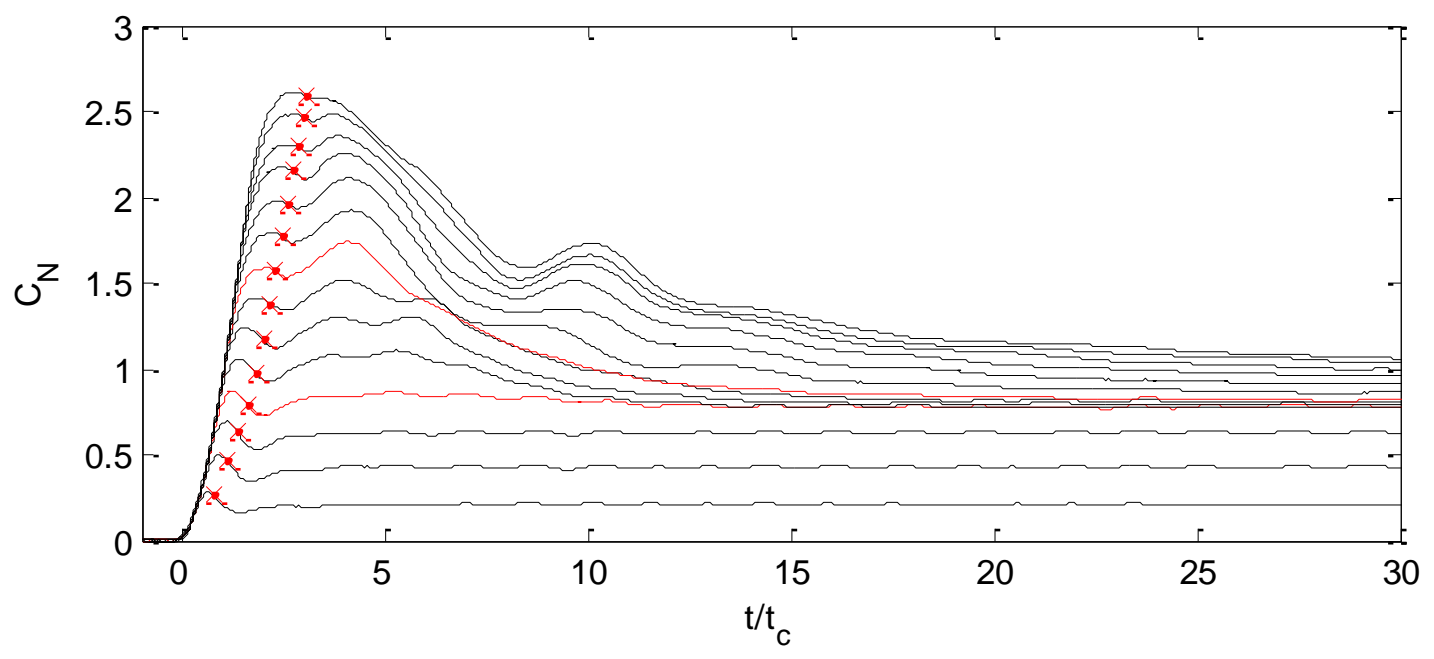

Fig. 7 Plate normal force coefficient of a trapezoidal plate as a function of normalized time for maximum angles of attack in the range $3-42$ in step increments of 3 degrees.

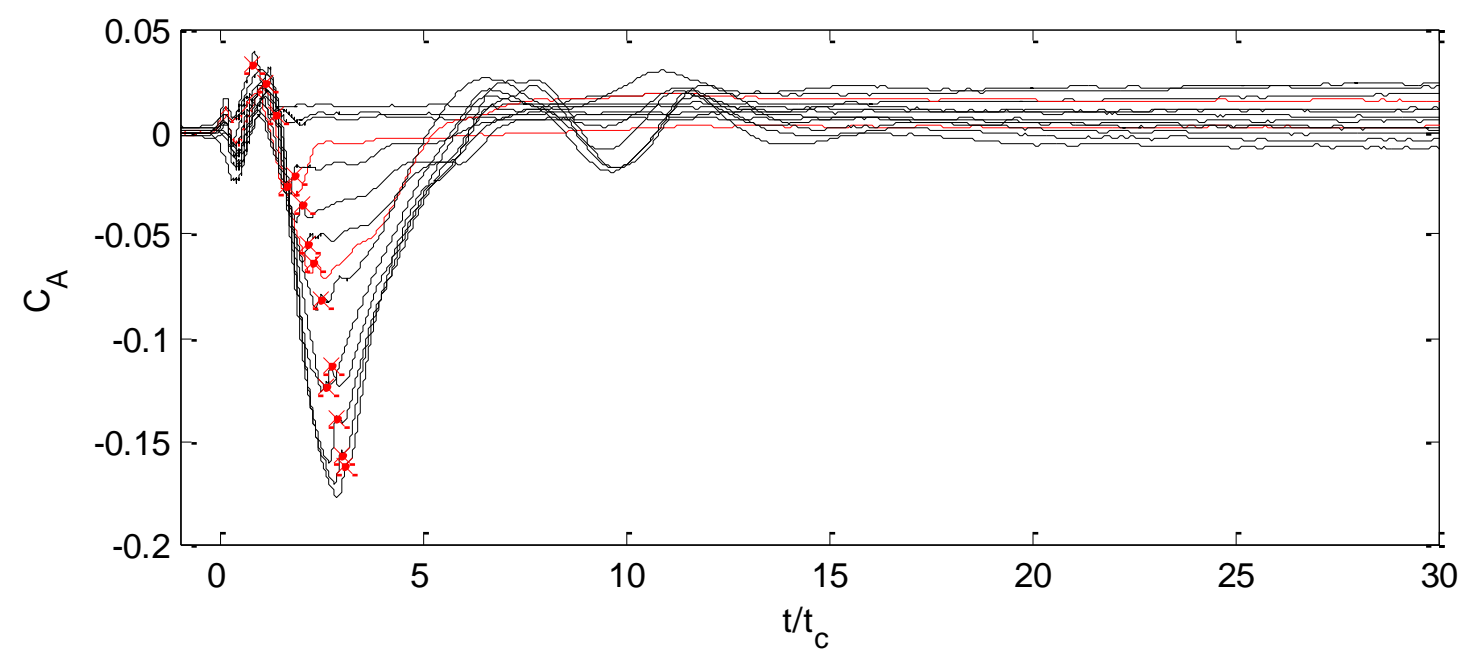

Fig. 8 Axial force coefficient of a trapezoidal plate as a function of normalized time for maximum angles of attack in the range 3 - 42 in step increments of 3 degrees.

The flow regions characterized from Figs. 7 - 8 are also presented in Figs. 9 - 10 for lift and drag coefficients. Figure 9 shows several maxima of lift coefficient: the first maximum during the wing motion, the second maximum at 3.8 convective times, and the third maximum around 10 convective times. These maxima are not perceived at steady state. The steady lift coefficient becomes less dependent on the angle of attack that is higher than 12 degrees, which is similar to normal force coefficient. Unlike spike-like lift coefficient during wing motion, the drag coefficient increases rapidly to a given maximum angle of attack with no force saturation. However, several transient maxima are still observed. Moreover, the steady drag coefficient increases aggressively with angle of attack, different from lift and normal force coefficients. 


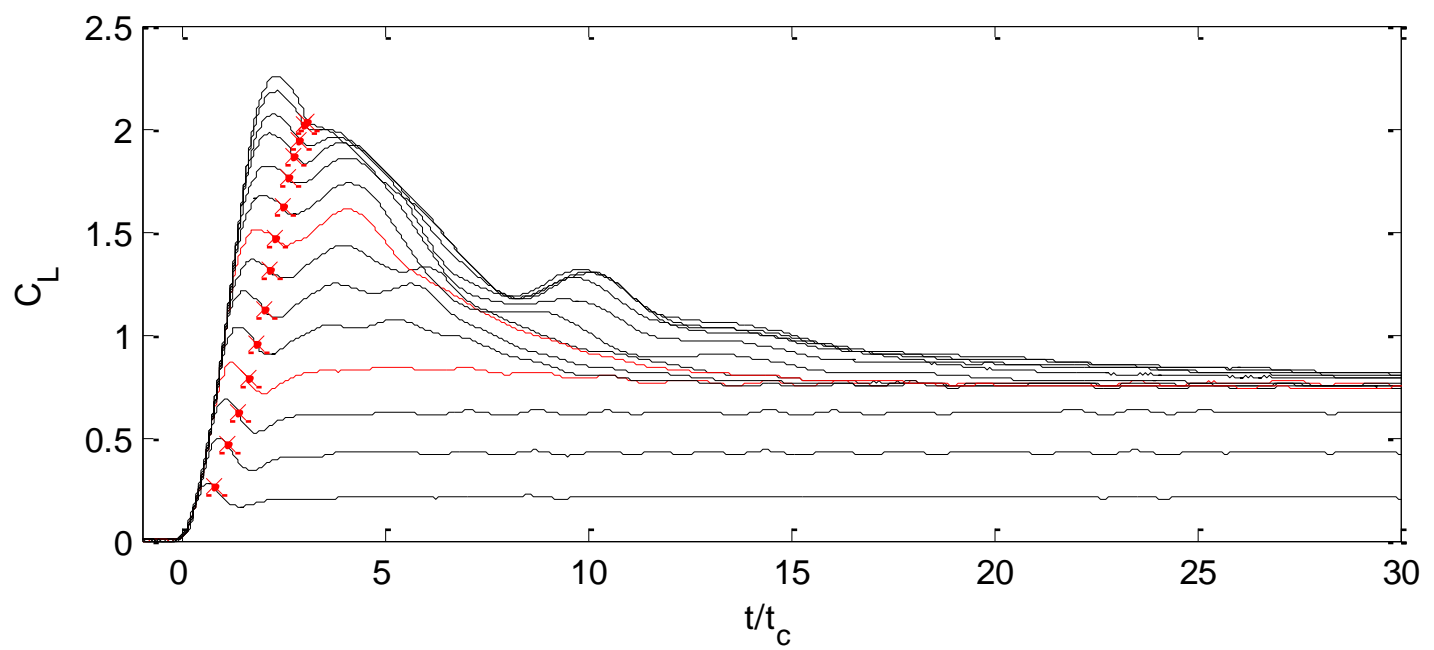

Fig. 9 Lift coefficient of a trapezoidal plate as a function of normalized time for maximum angles of attack in the range $3-42$ in step increments of 3 degrees.

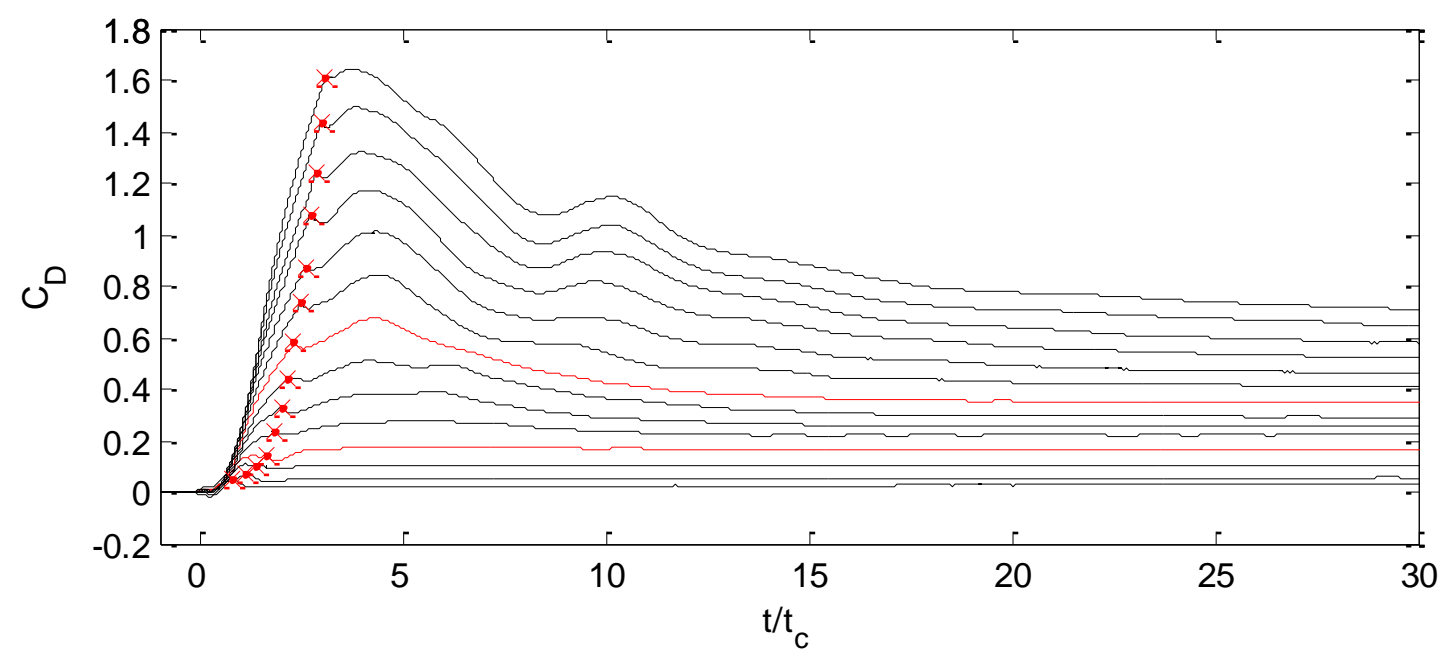

Fig. 10 Drag coefficient of a trapezoidal plate as a function of normalized time for maximum angles of attack in the range $3-42$ in step increments of 3 degrees.

\section{Force evolution of triangular wing}

Figures 11 through 14, for the triangular wing, show the plate normal, axial, lift and drag coefficients evolution at a function of time normalized by the convective time, successively. These data show a little difference from rectangular and trapezoidal wings. For the maximum angle of attack in the range 3 - 6 degrees, as shown in Fig. 11, the normal force coefficient reaches a maximum within the wing motion, and then decreases, even after the wing stops, until 1.5 convective times. Then a gradual increase follows. On the way to a steady state value at 30 convective times, the wing apparently experiences some oscillations. For the maximum angle of attack in the range $9-15$ degrees, the first maximum that occurs during the wing motion increases with maximum angle of attack. There is a second maximum grows at 2.8 convective times, less sensitive to the change of angle of attack. The second maxima have values lower than the first maxima, different from the characteristics of rectangular and trapezoidal wings discussed in previous sections. Toward steady state show some oscillations, similar to that at a lower angle of attack. For the maximum angle of attack in the range 18 - 27 degrees, the first maximum remains positive correlation with a maximum angle of attack and has values higher than the second maximum. However, the second maximum starts to develop at 3.8 convective times instead of 2.8 convective times as the angle of attack is increased. A third maximum is likely to form after 5 convective times, followed by a gradual decrease to a steady state value. For the maximum angle of attack 
in the range 30 - 36 degrees, the first maximum remains the same characteristics as that for the lower maximum angle of attack cases, i.e. its occurrence before the wing stops and its value increased with the maximum angle of attack. The second and third maxima merge at 3.8 convective times. A gradual decrease follows until 30 convective times; no other oscillations are determinable. For maximum angles of attack 39 - 42 degrees, the first maximum exists and the normal force coefficient decreases to steady state value with no oscillation. Figure 12 shows the first minimum axial force coefficient is in phase with the first maximum of normal force coefficient. The transient maxima of normal force coefficient found are hardly correlated with axial force coefficient after the wing motion stops. In addition, the amplitude of axial force is tenfold smaller than normal force coefficient.

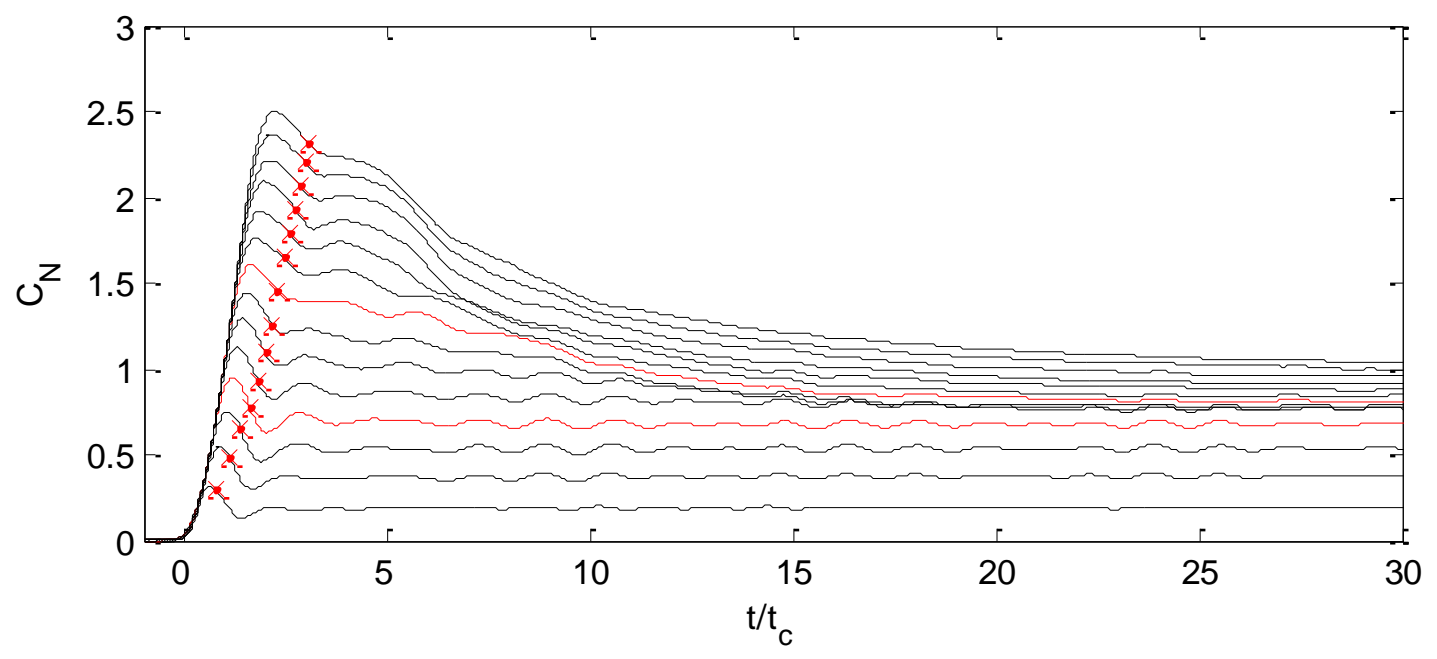

Fig. 11 Plate normal force coefficient of a triangular plate as a function of normalized time for maximum angle of attack in the range $3-42$ in step increments of 3 degrees.

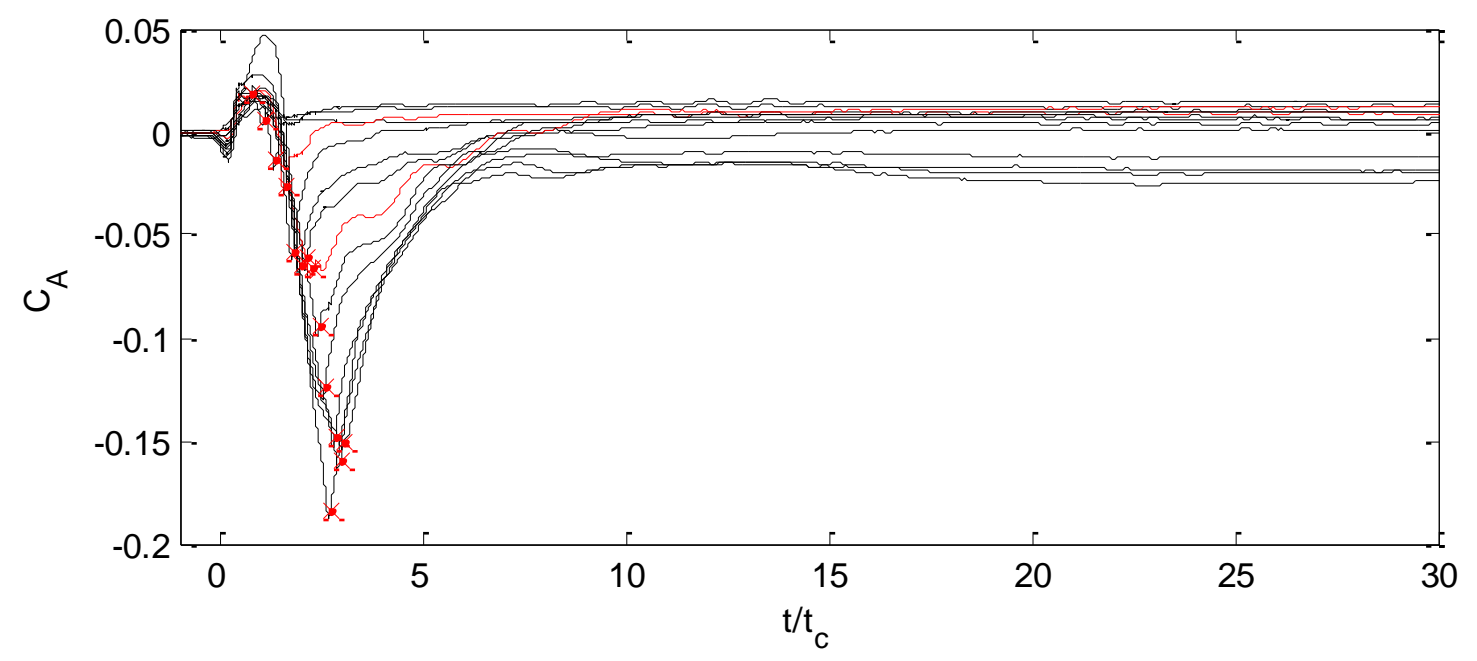

Fig. 12 Axial force coefficient of a triangular plate as a function of normalized time for maximum angles of attack in the range $3-42$ in step increments of 3 degrees.

The lift coefficient of triangular wing shown in Fig. 13 inherits similar characteristics from normal force coefficient, which is the same feature as that of rectangular and trapezoidal wings. The drag coefficient, shown in Fig. 14, increase rapidly but not as linear as that of rectangular and trapezoidal wings for the maximum angle of attack higher than 12 degrees. Less transient maxima are presented except at 2.8 and 3.8 convective times. 


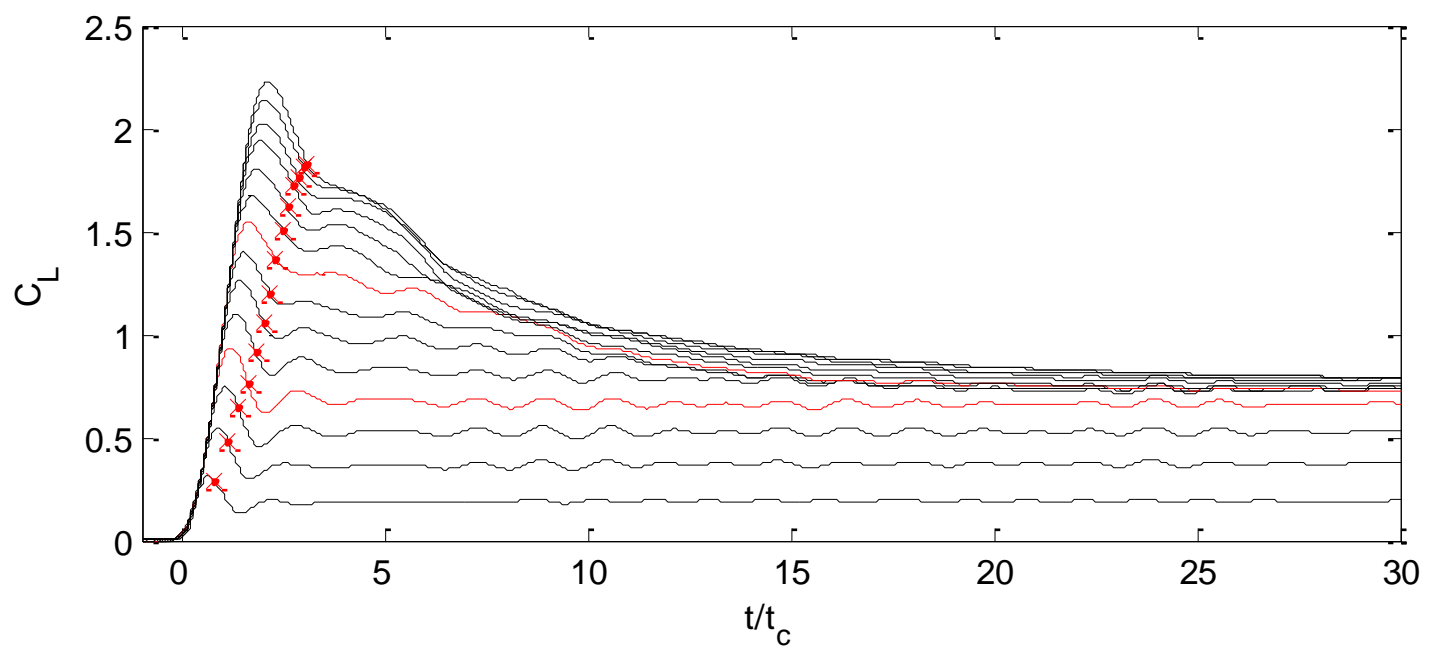

Fig. 13 Lift coefficient of a triangular plate as a function of normalized time for maximum angles of attack in the range $3-42$ in step increments of 3 degrees.

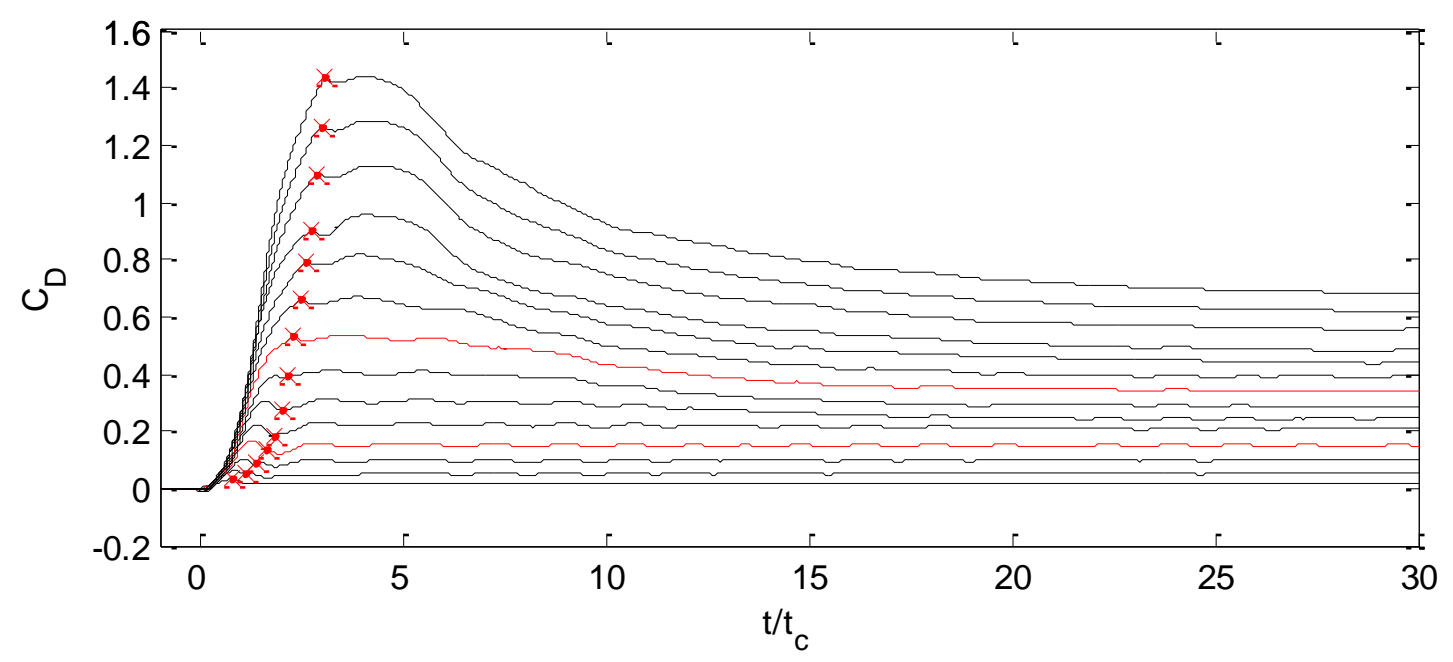

Fig. 14 Drag coefficient of a triangular plate as a function of normalized time for maximum angles of attack in the range $3-42$ in step increments of 3 degrees.

\section{Unsteady forces vs. steady forces with respect to maximum angle of attack}

Here the comparisons of unsteady force and steady force as a function of angle of attack are given in Figs. 15-16. The unsteady force is the measured force as the wing right stops at a given angle of attack, shown as empty symbols in Figs. 15-16. The unsteady force is denoted by red cross symbols in Figs. 5-6, 9-10, 13-14. The steady force is the measured force at 30 convective times, shown as a gray filled symbol. The measured force of rectangular, trapezoidal, and triangular wings are distinguished using square, diamond, and triangle marker, respectively. Several features are observed. First, the steady lift force saturates after 12 degrees whereas the unsteady lift force increases continuously with the angle of attack. The increase of unsteady lift coefficient slows down as the angle of attack is higher than 30 degrees. The phenomena of steady lift saturation are unobvious for steady drag force at the angle of attack higher than 12 degrees, in turn, yielding the unsteady drag force higher than steady values. The amount of deviation of unsteady drag force from steady value grows with the angle of attack. Second, it is intriguing that both rectangular and trapezoidal wings give close unsteady lift and drag. However, both trapezoidal and triangular wings give close steady forces. Similar behaviors are also reported by [8] for pitching wings at a constant rate. 

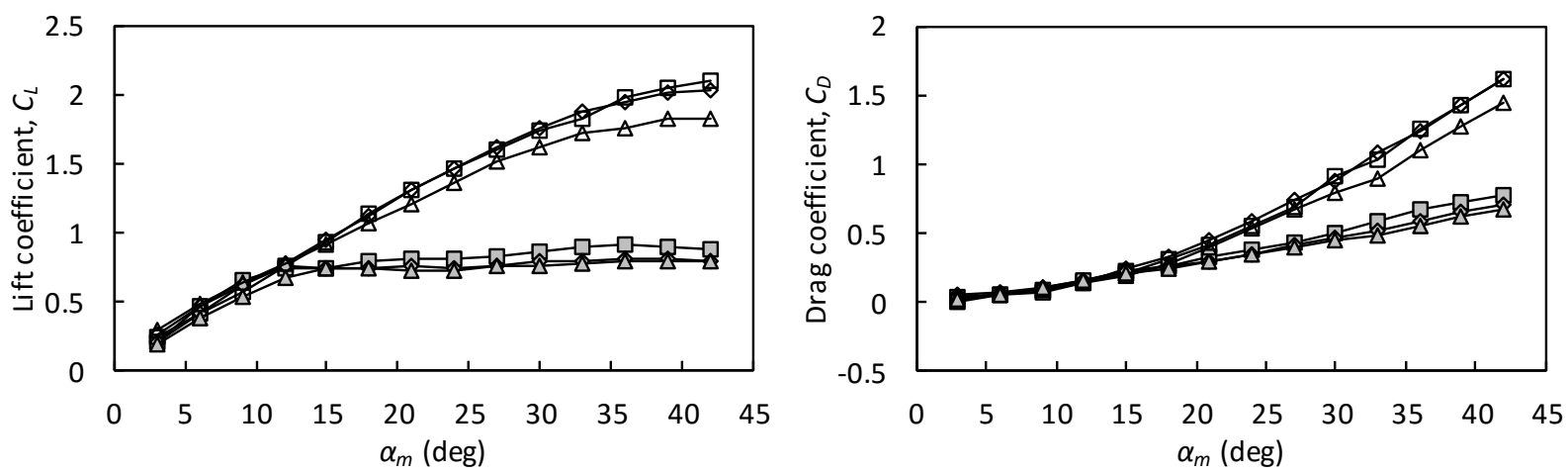

Fig. 15 Comparisons of unsteady and steady forces as a function of angle of attack among wing planform variation: rectangle (square symbol), trapezoid (diamond symbol), and triangle (triangle symbol).

Figure 16 shows the ratio of lift coefficient to drag coefficient as a function of angle of attack. The variations of both unsteady and steady $C_{L} / C_{D}$ are large at lower angles of attack in this parameter space. Surprisingly, these variations become smaller as the angle of attack is increased. The ratio $C_{L} / C_{D}$ converges to a value of one when the angle of attack is close to 45 degrees.

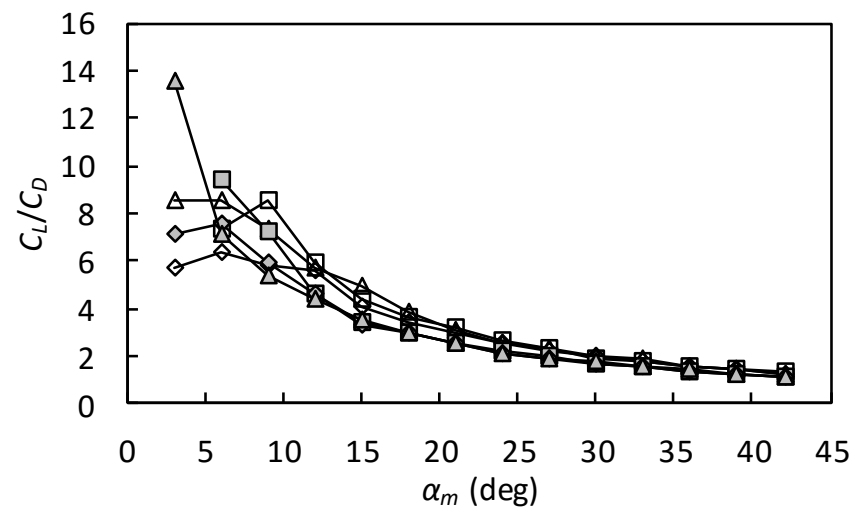

Fig. 16 The unsteady (empty symbol) and steady (gray filled symbol) ratio of $C_{L}$ to $C_{D}$ with respect to angle of attack among various wing planforms.

Figure 17 presents the characteristics of maximum unsteady lift coefficient occurs within the wing motion before the maximum angle of attack. These maximum lift coefficients occur after a half pitching time $0.5 t_{p}$ and have values higher than those values at the inception of transient force at the maximum angle of attack. The left panel of Fig. 17 shows the variation of maximum lift coefficient with angle of attack among wing planforms as it is scaled with instant lift coefficient at the maximum angle of attack. However, such variation of maximum lift coefficient is insignificant among various planforms in the original scale.
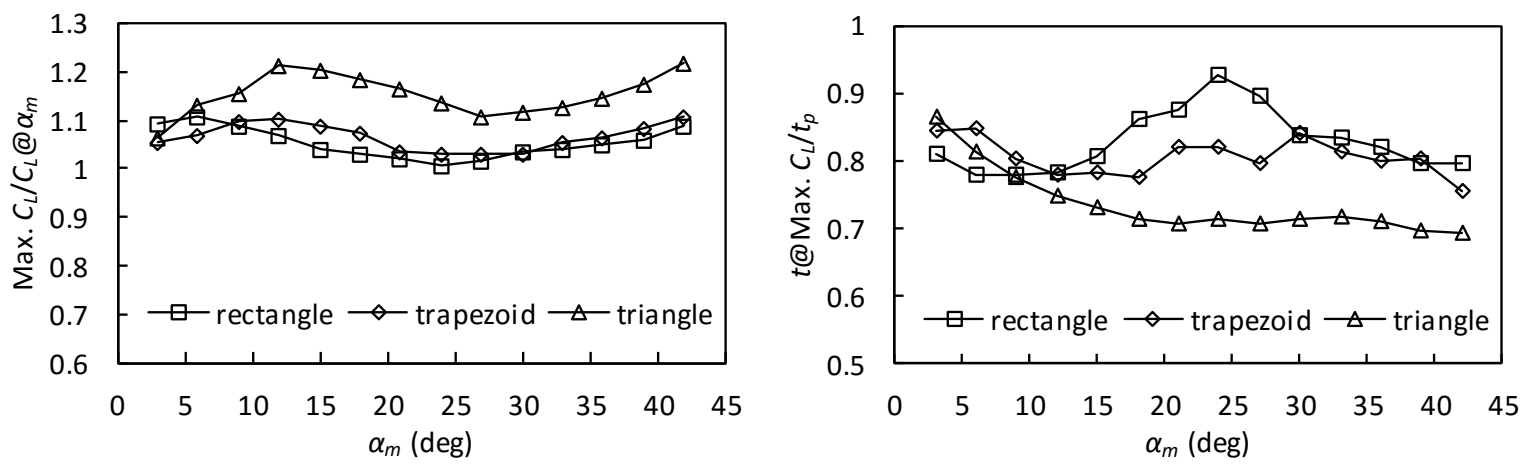

Fig. 17 Characteristics of maximum lift coefficient during wing pitching-up phase. 


\section{E. Strouhal number analysis}

To characterize shedding flow over bluff bodies, Strouhal number $S t$ is commonly suggested and employed here to study the behaviors of oscillatory force. The Strouhal number is defined by

$$
S t=f L / U_{\infty}
$$

where $f$ is the averaged frequency and determined from two distinct successive peaks of normal force coefficient. $U_{\infty}$ is the free stream velocity $(=17.5 \mathrm{~cm} / \mathrm{s})$. $L$ is the projected chord length $c \sin \alpha_{m}$. Figure 18 shows the Strouhal number found at several maximum angles of attack before steady state. For the maximum angle of attack lower than 21 degrees, in spite of planform variation, the wings yield Strouhal number following the similar tendency of development with angles of attack. For the maximum angle of attack higher than 24 degrees, the Strouhal number of triangular wing keeps increasing on the same path until 27 degrees; no more oscillatory force is observed beyond 27 degrees. However, the Strouhal numbers of rectangular and trapezoidal wings drop to 0.12 and 0.09 , respectively, and are less dependent on the increase of maximum pitch angle until 39 degrees. Both Strouhal numbers are less than 0.2 reported by White [10] for shedding flow over a circular cylinder under the same free steam $R e=8,900$. At 42-degree angle of attack, successive peaks of normal force are insignificant, resulting in no oscillation frequency identified. Additionally, Fig. 18 indicates two separate vortex dynamic mechanisms. The first mechanism would be associated with the type of Kármán vortex shedding for lower angles of attack. The second mechanism is associated with the alternate formation of leading edge vortex and trailing edge vortex, as has been revealed in our previous work for reduced pitch rate $K=$ 0.065 using 2D PIV system [7] and lens-shifted stereo PIV system [9]. The later vortex mechanism yields no shedding behavior; the vortex structures dissipate at the forming edge in turn.

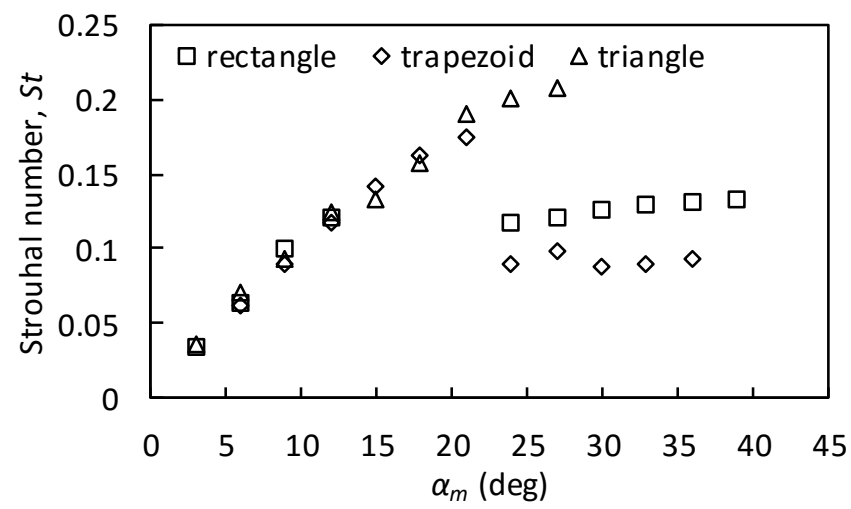

Fig. 18 Strouhal number of transient force oscillation among different planform wings.

\section{IV.Conclusions}

In this study, the rapidly pitching finite wings at mid-chord pivot were investigated using a constant pitch acceleration by means of direct force measurement for the better understanding of force generation mechanism at given angles of attack from 3 to 42 degrees. Concluding remarks are listed below.

1. Normal force is tenfold larger than axial force and therefore gives the most contribution to lift and drag forces.

2. During the wing motion of all angles of attack of interest, a maximum normal force is generated and its amplitude increases with the increase of the maximum angle of attack specified. This maximum normal force is correlated with a minimum axial force with a negative value. This phenomenon suggests the attached and pressure-dominated flow.

3. During the relaxation phase at a given maximum angle of attack, both normal force and axial force of rectangular and trapezoidal wings are excited more easily to yield the course of transient oscillation. For the angle of attack higher than 24 degrees, the Strouhal number is scattered around 0.1 that is different from the increasing Strouhal number (up to 0.2) with the maximum angles of attack lower than 24 degrees. 


\section{Acknowledgments}

This work was supported by Ministry of Science and Technology of Taiwan, R.O.C., under Contract MOST 1052221-E-606-007.

\section{References}

[1] Ol, M. V., and Babinsky, H., "Unsteady Flat Plates: A Cursory Review of AVT-202 Research (Invited)," AIAA Paper 2016-0285, Jan. 2016.

-[2] Stevens, P. R. R. J., Babinsky, H., Manar, F., Mancini, P., Jones, A. R., Granlund, K. O., Ol, M. V., Nakata, T., Phillips, N., Bomphrey, R. J., and Gozukara, A. C., "Low Reynolds Number Acceleration of Flat Plate Wings at High Incidence (Invited)," 54th AIAA Aerospace Sciences Meeting, AIAA Paper 2016-0286, Jan. 2016.

-[3] Bernal, L. P., "Unsteady Aerodynamics of Pitching Low Aspect Ratio Wings: A review of AVT202 Panel Results (Invited)," 54th AIAA Aerospace Sciences Meeting, AIAA Paper 2016-0287, Jan. 2016.

-[4] Jones, A. R., Manar, F., Philips, N., Nakata, T., Bomphrey, R. J., Ringuette, M., Percin, M., Oudheusden, B. V., and Palmer, J. L., "Leading-Edge Vortex Evolution and Lift Production on Rotating Wings," 54th AIAA Aerospace Sciences Meeting, AIAA Paper 2016-0288, Jan. 2016.

-[5] Son, O., Cetiner, O., Stevens, P. R. R. J., Babinsky, H., Manar, F., Mancini, P., Jones, A. R., Ol, M. V., and Gozukara, A. C., "Parametric Variations in Aspect Ratio, Leading Edge and Planform Shapes for the Rectilinear Pitch Cases of AVT-202," 54th AIAA Aerospace Sciences Meeting, AIAA Paper 2016-0289, Jan. 2016.

-[6] Yu, H.-T., and Bernal, L. P., "Effect of Pivot Point on Aerodynamic Force and Vortical Structure of Pitching Flat Plate Wings," 51st AIAA Aerospace Sciences Meeting, AIAA Paper 2013-0792, Jan. 2013.

[7] Yu, H.-T., and Bernal, L.P., "Effects of Pivot Location and Reduced Pitch Rate on Pitching Rectangular Flat Plates," AIAA Journal, (2013), accessed April 15, 2013. doi: http://arc.aiaa.org/doi/abs/10.2514/1.J055244.

-[8] Yu, H.-T., Bernal, L.P., and Ol, M.V., "Effects of Planform Geometry and Pivot Axis Location on the Aerodynamics of Pitching Low Aspect Ratio Wings," 43rd Fluid Dynamics Conference, AIAA Paper 2013-2992, June 2013.

[9] Yu, H.-T., "Low Reynolds Number Aerodynamics of Finite Wing at Low Reduced Pitch Rates," Journal of Aeronautics, Astronautics and Aviation, Vol. 48, No. 2, pp. 83-94, 2016.

[10]White, F.M., Viscous Fluid Flow, $3^{\text {rd }}$ ed., McGraw-Hill, New York, 2006, pp. 11. 
This article has been cited by:

1. Huai-Te Yu, Luis P. Bernal. Transient Force Evolution of Swept Flat-Plate Wings Pitching at a Constant Acceleration Amplitude . [Citation] [PDF] [PDF Plus] 